Journal of Computational and Applied Mechanics, Vol. 11., No. 1., (2016), pp. 49-66 DOI: $10.32973 /$ jcam.2016.004

\title{
HYPERSINGULAR BOUNDARY INTEGRAL FORMULATIONS FOR PLANE ELASTICITY IN TERMS OF FIRST-ORDER STRESS FUNCTIONS
}

\author{
SÁNDOR SZIRBIK \\ Institute of Engineering Mechanics, University of Miskolc \\ H-3515 Miskolc, Miskolc-Egyetemváros, Hungary \\ sandor.szirbik@uni-miskolc.hu \\ [Received: February 26, 2016. Accepted: April 25, 2016]
}

\begin{abstract}
In this paper the hypersingular integral equation method in the dual system of plane elasticity is developed and applied to mixed boundary value problems providing that the region under consideration is simply connected. The accuracy of stress calculations on the boundary is greatly increased if one applies hypersingular integral equations instead of using the traditional computational techniques of the boundary element method. In contrast to earlier results which formulate the hypersingular integral equations in the primal system of elasticity, we attack the plane problems in a dual formulation in which the stress functions of order one and the rigid body rotation are the fundamental variables. It is a further advantage of the dual formulation that the stress components can be obtained directly from the stress functions of order one. There is therefore no need for Hooke's law, which should be used when we determine the stress components on the boundary from displacements. The numerical examples presented prove the efficiency of this technique.
\end{abstract}

Mathematical Subject Classification: 74S15, 45F15

Keywords: Dual formulation, stress functions of order one, strain boundary conditions, hypersingular formulation in the dual system

\section{INTRODUCTION}

The theory of the boundary integral equations with hypersingular kernels in the primal system of elasticity is well developed and there are a number of problems these include some problems of fracture mechanics - for the solution of which they are successfully applied. Guiggiani at al. [1] is, in all probability, one of the first papers devoted to this issue. The authors came to the conclusion that a deep analysis of the hypersingular formulation provides an opportunity to increase the accuracy of the stress components computed at interior points and points on the contour. Later on this technique was developed further for $2 \mathrm{D}$ and $3 \mathrm{D}$ problems in the primal system of elasticity 2,4$]$. 
The boundary integral equations of the direct method in the dual system of plane elasticity are established in [5] and [6]. These works formulate the dual boundary integral equations for plane problems in terms of stress functions of order one and the rigid body rotation.

The paper is organized into six sections. Section 2 outlines the most important preliminaries, i.e., it presents the dual integral equations of the direct method and clarifies what the boundary conditions are for the stress functions. The concept of the strain boundary conditions is also introduced. When using the direct boundary element method one has to compute weakly and strongly singular integrals. This is the main reason for obtaining partly inaccurate results for the stresses at points close to the boundary. Surprisingly the efficiency of the numerical solutions could be increased by using an appropriately chosen hypersingular formulation 1, 2]. Section 3 deals with the issue of how to introduce the hypersingular integral formulation for those boundary value problems in which the governing equations are given in terms of stress functions of order one. The necessary equations are set up in a suitable form in Section 4 which is devoted to some important computational issues so that we can develop an appropriate algorithm for the stress computations. Section 5 presents the applicability of the algorithm for a simple and a bit more difficult problem. The last section is a summary of the results. Some longer formulae are presented in Appendix A.

\section{Preliminaries}

Throughout this paper $x_{\rho}$ and $y_{\rho}$ are rectangular Cartesian coordinates referred to the same origin $O$. (Greek)[Latin] subscripts are assumed to have the range $(1,2)[1,2,3]$. For subscripts repeated twice, the summation convention is applied. In accordance

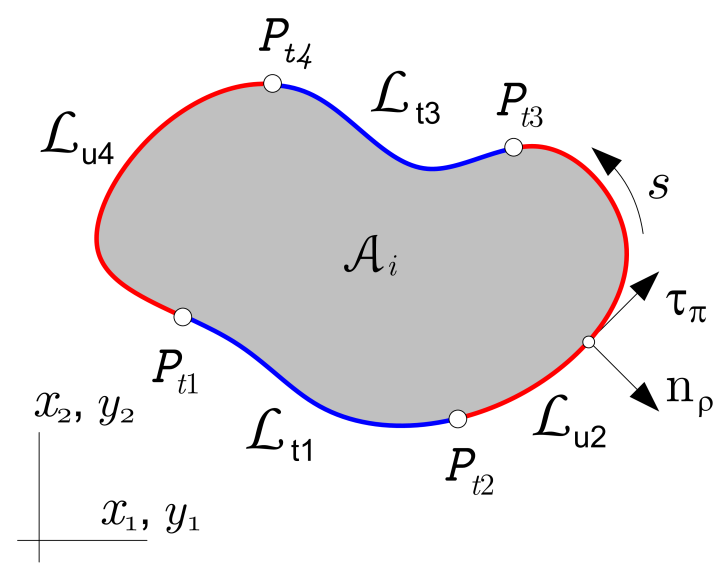

Figure 1. The inner region $\mathcal{A}_{i}$ 
with the notations introduced, $\delta_{\rho \lambda}$ is the Kronecker symbol, derivatives with respect to $y_{\rho}$ are denoted by the subscript $\rho$ preceded by a coma and $\epsilon_{\rho \pi 3}$ is the permutation symbol.

Consider a simply connected inner region $\mathcal{A}_{i}$. The contour $\mathcal{L}_{o}$ of the region can be divided into two parts denoted by $\mathcal{L}_{t}$ and $\mathcal{L}_{u}$. We shall assume that $\left[\mathcal{L}_{t}\right]\left\{\mathcal{L}_{u}\right\}$ is the union of those arcs on which [stress functions (obtainable from prescribed tractions)] \{strain boundary conditions (derivatives of displacements with respect to the arc coordinate) $\}$ are imposed. If the number of arcs on the boundary is four they are denoted by $\mathcal{L}_{t 1}, \mathcal{L}_{t 3}$ and $\mathcal{L}_{u 2}, \mathcal{L}_{u 4}$, respectively. The quantities that are prescribed on the boundary are denoted by hatted letters: $\hat{u}_{\lambda}$ and $\hat{t}_{\lambda}$ are the displacements and stresses (tractions) prescribed on the boundary. Let $y=\left(y_{1}, y_{2}\right)$ and $x=\left(x_{1}, x_{2}\right)$ be the source point and field point.

The three dual Somigliana formulae for the inner region are of the form

$$
\begin{aligned}
& \mathfrak{u}_{k}(y)=\oint_{\mathcal{L}_{o}} \mathfrak{U}_{k \lambda}(x, y) \mathfrak{t}_{\lambda}(x) \mathrm{d} s_{x}-\oint_{\mathcal{L}_{o}} \mathfrak{T}_{k \lambda}(x, y) \mathfrak{u}_{\lambda}(x) \mathrm{d} s_{x} \quad y \in \mathcal{A}_{i}, \\
& c_{\kappa \lambda}(y) \mathfrak{u}_{\lambda}(y)=\oint_{\mathcal{L}_{o}} \mathfrak{U}_{\kappa \lambda}(x, y) \mathfrak{t}_{\lambda}(x) \mathrm{d} s_{x}-\oint_{\mathcal{L}_{o}} \mathfrak{T}_{\kappa \lambda}(x, y) \mathfrak{u}_{\lambda}(x) \mathrm{d} s_{x} \quad y \in \mathcal{L}_{o}, \\
& 0=\oint_{\mathcal{L}_{o}} \mathfrak{U}_{\kappa \lambda}(x, y) \mathfrak{t}_{\lambda}(x) \mathrm{d} s_{x}-\oint_{\mathcal{L}_{o}} \mathfrak{T}_{\kappa \lambda}(x, y) \mathfrak{u}_{\lambda}(x) \mathrm{d} s_{x} \quad y \notin \mathcal{A}_{i} \cup \mathcal{L}_{o},
\end{aligned}
$$

in which the stress functions of order one $\mathfrak{u}_{\lambda}$ - this vector is also referred to as dual displacement vector - and the rigid body rotation $\mathfrak{u}_{3}=\varphi$ constitute the vector $\mathfrak{u}_{k}$,

$$
\left[\mathfrak{U}_{k l}(x, y)\right]=\mathfrak{K}_{1}\left[\begin{array}{ccc}
-2 \ln R-3-2 \frac{r_{2} r_{2}}{R^{2}} & 2 \frac{r_{1} r_{2}}{R^{2}} & \frac{2}{\mu}(1-\nu) \frac{r_{1}}{R^{2}} \\
2 \frac{r_{2} r_{1}}{R^{2}} & -2 \ln R-3-2 \frac{r_{1} r_{1}}{R^{2}} & \frac{2}{\mu}(1-\nu) \frac{r_{2}}{R^{2}} \\
\frac{2}{\mu}(1-\nu) \frac{r_{1}}{R^{2}} & \frac{2}{\mu}(1-\nu) \frac{r_{2}}{R^{2}} & 0
\end{array}\right],
$$

is the fundamental solution of order one, the distance between the points $x_{\rho}$ and $y_{\rho}$ is $R$, the position vector is defined as $r_{\rho}=x_{\rho}-y_{\rho}, s$ is the arc coordinate on $\mathcal{L}_{o}$ (the positive direction on $\mathcal{L}_{o}$ is the one which leaves the region on the left side), $\mathfrak{t}_{\lambda}(x)$ stands for the derivative $-d u_{\lambda} / d s$ taken on the boundary (here $u_{\lambda}$ is the displacement vector) - this quantity is also referred to as the dual stress vector,$- \mu$ and $\nu$ are the shear modulus of elasticity and the Poisson number, respectively, 


$$
\left[\mathfrak{T}_{l \lambda}(x, y)\right]=\mathfrak{D}(R)\left[\begin{array}{cc}
n_{1} r_{1}\left(4 \frac{r_{2}^{2}}{R^{2}}-2(3-2 v)\right) & -n_{2} r_{1}\left(4 \frac{r_{2}^{2}}{R^{2}}+2(1-2 v)\right) \\
+n_{2} r_{2}\left(4 \frac{r_{2}^{2}}{R^{2}}-2(3-2 v)\right) & -n_{1} r_{2}\left(4 \frac{r_{1}^{2}}{R^{2}}-2(1-2 v)\right) \\
-n_{1} r_{2}\left(4 \frac{r_{1}^{2}}{R^{2}}+2(1-2 v)\right) & n_{2} r_{2}\left(4 \frac{r_{1}^{2}}{R^{2}}-2(3-2 v)\right) \\
-n_{2} r_{1}\left(4 \frac{r_{2}^{2}}{R^{2}}-2(1-2 v)\right) & +n_{1} r_{1}\left(4 \frac{r_{1}^{2}}{R^{2}}-2(3-2 v)\right) \\
-n_{1} \frac{2}{\mu}(1-\nu) \frac{r_{1}^{2}-r_{2}^{2}}{R^{2}} & -n_{1} \frac{4}{\mu}(1-\nu) \frac{r_{1} r_{2}}{R^{2}} \\
-n_{2} \frac{4}{\mu}(1-\nu) \frac{r_{1} r_{2}}{R^{2}} & +n_{2} \frac{2}{\mu}(1-\nu) \frac{r_{1}^{2}-r_{2}^{2}}{R^{2}}
\end{array}\right]
$$

is the fundamental solution of order two, $n_{\rho}$ is the outer unit normal, $c_{\kappa \lambda}(y)$ depends on the angle formed by the tangents to the contour at $y\left(c_{\kappa \lambda}(y)=\delta_{\kappa \lambda} / 2\right.$ if the contour is smooth at $y) . \mathfrak{U}_{\kappa \lambda}(x, y)$ and $\mathfrak{T}_{\kappa \lambda}(x, y)$ are weakly and strongly singular. Integral equation (2.1b) is that of the direct method for inner regions in the dual system of plane elasticity: the unknowns are $\mathfrak{u}_{\lambda}(x)$ on $\mathcal{L}_{u}$ and $\mathfrak{t}_{\lambda}(x)$ on $\mathcal{L}_{t}$,5, 6. The line integrals in $2.1 \mathrm{~b}$ ) should be taken in principal value.

Equation 2.1a) should be associated with appropriate boundary conditions. One can readily check that the stress function vector (the dual displacement vector) on the $\operatorname{arcs}$ of $\mathcal{L}_{t i}$ is of the form

$$
\hat{\mathfrak{u}}_{\rho}(s)=\int_{P_{t i}}^{s} \hat{t}_{\rho}(\sigma) \mathrm{d} \sigma \quad s \in \mathcal{L}_{t i} \quad i=1,3,
$$

where - in accordance with all that has been said earlier - the hatted letters denote the prescribed values. Consequently, equation

$$
\mathfrak{u}_{\rho}(s)=\hat{\mathfrak{u}}_{\rho}(s)+C_{\rho}^{(t i)} \quad s \in \mathcal{L}_{t i} \quad i=1,3
$$

is the traction boundary condition. Observe that the quantities $C_{\rho}^{(t i)}$ in equation 2.5 are undetermined constants of integration.

The so-called strain boundary conditions on the arcs on $\mathcal{L}_{u i}$ have the following form:

$$
\frac{\mathrm{d} \hat{u}_{\lambda}}{\mathrm{d} s}=n_{\rho}\left[\epsilon_{\rho \pi 3} e_{\pi \lambda}-\delta_{\rho \lambda} \varphi_{3}\right] \quad s \in \mathcal{L}_{u i} \quad i=2,4
$$

where $\hat{u}_{\lambda}(s)$ is the vector of the prescribed displacements on $\mathcal{L}_{u i}$ and $e_{\kappa \lambda}$ are the in-plane components of the strain tensor.

If tractions (stress functions) are imposed on the whole contour $\mathcal{L}_{o}\left(\mathcal{L}_{t} \equiv \mathcal{L}_{o}\right)$, the compatibility condition in the large

$$
\int_{\mathcal{L}_{o}} n_{\rho}\left[\epsilon_{\rho \pi 3} e_{\pi \lambda}-\delta_{\rho \lambda} \varphi_{3}\right] \mathrm{d} s=0
$$


should also be satisfied. If tractions (stress functions) are imposed only on the arcs $\mathcal{L}_{t 1}, \mathcal{L}_{t 3}$ of the contour $\mathcal{L}_{o}$, the supplementary conditions of single valuedness to be satisfied are

$$
\int_{\mathcal{L}_{t i}} n_{\rho}\left[\epsilon_{\rho \pi 3} e_{\kappa \lambda}-\delta_{\rho \lambda} \varphi_{3}\right] \mathrm{d} s-\left.\hat{u}_{\lambda}\right|_{P_{t i}} ^{P_{t, i+1}}=0 \quad i=1,3,
$$

where the extremities of the $\operatorname{arc} \mathcal{L}_{t i}$ are denoted by $P_{t i}$ and $P_{t, i+1}$, respectively.

If the compatibility field equation

$$
\epsilon^{\kappa \rho 3} e_{\lambda \kappa ; \rho}+\varphi_{. ; \lambda}^{3}=\epsilon^{\kappa \rho 3}\left(e_{\lambda \kappa ; \rho}-\epsilon_{\lambda \kappa 3} \varphi_{. ; \rho}^{3}\right)=0 \quad x \in A_{i}
$$

is fulfilled then the supplementary compatibility conditions 2.8 are not independent - one can be omitted 7]. Since no stresses belong to constant stress functions, we can also set two constants (one vector $C_{\rho}^{(t i)}$ ) to zero .

In this way, we have as many independent conditions of single valuedness $(2.8)$ as there are undetermined integration constants $C_{\rho}^{(t i)}$ in the traction boundary condition 2.5.

The outer region $A_{e}$ is understood as the part of the coordinate plane outside the closed curve $\mathcal{L}_{o}$ - see Figure 1 . We shall assume that the stresses at infinity denoted by

$$
t_{11}(\infty), t_{12}(\infty)=t_{21}(\infty), t_{22}(\infty)
$$

are all constants. We shall also assume that the rigid body rotation vanishes at infinity:

$$
\varphi_{3}(\infty)=0
$$

The stresses at infinity can be obtained from the stress functions

$$
\tilde{\mathfrak{u}}_{\lambda}(x)=\epsilon_{\alpha 3 \rho} x_{\alpha} t_{\lambda \rho}(\infty)+c_{\lambda}(\infty),
$$

where there belong no stresses to the constant stress function vector $c_{\lambda}(\infty)$.

The three dual Somigliana formulae for the outer region are of the form

$$
\begin{aligned}
& \mathfrak{u}_{k}(y)=\tilde{\mathfrak{u}}_{\lambda}(y)+\oint_{\mathcal{L}_{o}} \mathfrak{U}_{k \lambda}(x, y) \mathfrak{t}_{\lambda}(x) \mathrm{d} s_{x}-\oint_{\mathcal{L}_{o}} \mathfrak{T}_{k \lambda}(x, y) \mathfrak{u}_{\lambda}(x) \mathrm{d} s_{x} \quad y \in \mathcal{A}_{e}, \\
& c_{\kappa \lambda}(y) \mathfrak{u}_{\lambda}(y)=\tilde{\mathfrak{u}}_{\lambda}(y)+\oint_{\mathcal{L}_{o}} \mathfrak{U}_{\kappa \lambda}(x, y) \mathfrak{t}_{\lambda}(x) \mathrm{d} s_{x}-\oint_{\mathcal{L}_{o}} \mathfrak{T}_{\kappa \lambda}(x, y) \mathfrak{u}_{\lambda}(x) \mathrm{d} s_{x} \quad y \in \mathcal{L}_{o}, \\
& 0=\tilde{\mathfrak{u}}_{\lambda}(y)+\oint_{\mathcal{L}_{o}} \mathfrak{U}_{\kappa \lambda}(x, y) \mathfrak{t}_{\lambda}(x) \mathrm{d} s_{x}-\oint_{\mathcal{L}_{o}} \mathfrak{T}_{\kappa \lambda}(x, y) \mathfrak{u}_{\lambda}(x) \mathrm{d} s_{x} \quad y \notin \mathcal{A}_{e} \cup \mathcal{L}_{o}
\end{aligned}
$$

\section{Hypersingular integral EQUATIONS IN THE DUAL SYSTEM OF PLANE ELASTICITY}

If we would like to use hypersingular equations in the dual system, the partial derivatives of equation 2.1c with respect to the coordinates $y_{\rho}$ of the source point $y$ should 
be known. In order to obtain appropriate formulae, a small neighborhood of the source point $y$ should be removed from the region of integration: this way we can avoid calculating singular integrals - the kernels are singular at the point $x=y$. Assume that the small circular domain $\mathcal{A}_{\epsilon}$ with the source point as its center is removed from the original region $\mathcal{A}_{i}$. Consequently, the new contour consists of two arcs (i) $\mathcal{L}_{\varepsilon}$ left from $\mathcal{L}_{o}$ after the removal of the small circle with radius $\varepsilon$ and (ii) the circular arc $s_{\epsilon}$.

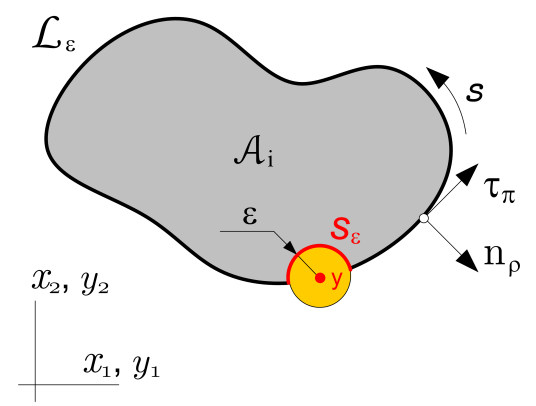

Figure 2. The boundary curve $\mathcal{L}_{\varepsilon} \cup s_{\epsilon}$

Under this condition equation 2.1c can be written in the form

$$
\begin{aligned}
0=\lim _{\varepsilon \rightarrow 0}\left\{\int _ { \mathcal { L } _ { \varepsilon } } \left[\mathfrak{U}_{k \lambda}(x, y) \mathfrak{t}_{\lambda}(x)\right.\right. & \left.-\mathfrak{T}_{k \lambda}(x, y) \mathfrak{u}_{\lambda}(x)\right] \mathrm{d} s_{x}+ \\
& \left.+\int_{s_{\varepsilon}}\left[\mathfrak{U}_{k \lambda}(x, y) \mathfrak{t}_{\lambda}(x)-\mathfrak{T}_{k \lambda}(x, y) \mathfrak{u}_{\lambda}(x)\right] \mathrm{d} s_{x}\right\} .
\end{aligned}
$$

After deriving equation (3.1) with respect $y_{\rho}$, we have

$$
\begin{aligned}
\lim _{\varepsilon \rightarrow 0}\left\{\int _ { \mathcal { L } _ { \varepsilon } } \left[\mathfrak{U}_{k \lambda, \rho}(x, y) \mathfrak{t}_{\lambda}(x)\right.\right. & \left.-\mathfrak{T}_{k \lambda, \rho}(x, y) \mathfrak{u}_{\lambda}(x)\right] \mathrm{d} s_{x}+ \\
& \left.+\int_{s_{\varepsilon}} \mathfrak{U}_{k \lambda, \rho}(x, y) \mathfrak{t}_{\lambda}(x)-\mathfrak{T}_{k \lambda, \rho}(x, y) \mathfrak{u}_{\lambda}(x) \mathrm{d} s_{x}\right\}=0
\end{aligned}
$$

where the derivatives $\mathfrak{U}_{k \lambda, \rho}$ are given below:

$$
\left.\mathfrak{U}_{k \lambda, 1}(x, y)\right]=\mathfrak{K}_{1}\left[\begin{array}{cc}
2 r_{1}-\frac{4 r_{1} r_{2}^{2}}{R^{2}} & -2 r_{2}+\frac{4 r_{1}^{2} r_{2}}{R^{2}} \\
-2 r_{2}+\frac{4 r_{1}^{2} r_{2}}{R^{2}} & 6 r_{1}-4 \frac{r_{1}^{3}}{R^{2}} \\
\frac{2}{\mu}(1-\nu) \frac{r_{1}^{2}-r_{2}^{2}}{R^{2}} & \frac{2}{\mu}(1-\nu) \frac{2 r_{1} r_{2}}{R^{2}}
\end{array}\right],
$$




$$
\left[\mathfrak{U}_{k \lambda, 2}(x, y)\right]=\mathfrak{K}_{1}\left[\begin{array}{cc}
6 r_{2}-\frac{4 r_{2}^{3}}{R^{2}} & -2 r_{1}+\frac{4 r_{1} r_{2}^{2}}{R^{2}} \\
-2 r_{1}+\frac{4 r_{1} r_{2}^{2}}{R^{2}} & 2 r_{2}-4 \frac{r_{1}^{2} r_{2}}{R^{2}} \\
\frac{2}{\mu}(1-\nu) \frac{2 r_{1} r_{2}}{R^{2}} & \frac{2}{\mu}(1-\nu) \frac{r_{2}^{2}-r_{1}^{2}}{R^{2}}
\end{array}\right] .
$$

We have also determined the derivatives $\mathfrak{T}_{\kappa \lambda, \rho}$. The results are presented in Appendix A - see equations A.1. Due to the derivation, the order of singularity of the kernels is increased, i.e., $\mathcal{D}_{k \rho \lambda}=\mathfrak{U}_{k \lambda, \rho}$ has a singularity of order one, while the singularity of $\mathcal{S}_{k \rho \lambda}=\mathfrak{T}_{k \lambda, \rho}$ is that of order two.

Assume further that the stress functions of order one are continuous and differentiable in the neighborhood of the point $y$. Then it holds with a good accuracy that

$$
\mathfrak{u}_{\lambda}(x)=\mathfrak{u}_{\lambda}(y)+\mathfrak{u}_{\lambda, \delta}(y)\left(x_{\delta}-y_{\delta}\right)+O\left(r^{1+\alpha}\right),
$$

where $O$ is the ordo symbol. It is also not too difficult to check that the displacement derivative $\mathfrak{t}_{\lambda}$ in the vicinity of the source point assumes the form

$$
\mathfrak{t}_{\lambda}(x)=-\frac{\partial u_{\lambda}}{\partial s} \cong-u_{\lambda, \pi}(y) \tau_{\pi}(x)=-u_{\lambda, \pi}(y) \epsilon_{3 \rho \pi} n_{\rho}(x),
$$

where the outward unit normal $n_{\rho}$ is taken at the point $x$ and $\tau_{\pi}$ is the unit tangent.

Making use of these approximations, we can rewrite equation $(3.2)$ in the form

$$
\begin{array}{r}
\lim _{\varepsilon \rightarrow 0}\left\{\int_{\mathcal{L}_{\varepsilon}}\left[\mathcal{D}_{k \rho \lambda} \mathfrak{t}_{\lambda}(x)-\mathcal{S}_{k \rho \lambda} \mathfrak{u}_{\lambda}(x)\right] \mathrm{d} s_{x}+\int_{s_{\varepsilon}} \mathcal{D}_{k \rho \lambda}\left[\mathfrak{t}_{\lambda}(x)+u_{\lambda, \pi}(y) \tau_{\pi}(x)\right] \mathrm{d} s_{x}-\right. \\
-\int_{s_{\varepsilon}} \mathcal{S}_{k \rho \lambda}\left[\mathfrak{u}_{\lambda}(x)-\mathfrak{u}_{\lambda, \delta}(y)\left(x_{\delta}-y_{\delta}\right)-\mathfrak{u}_{\lambda}(y)\right] \mathrm{d} s_{x}-\int_{s_{\varepsilon}} \mathcal{S}_{k \rho \lambda} \mathrm{d} s_{x} \mathfrak{u}_{\lambda}(y)- \\
\left.\quad-\int_{\mathcal{s}_{\varepsilon}} \mathcal{D}_{k \rho \lambda} u_{\lambda, \pi}(y) \tau_{\pi}(x) \mathrm{d} s_{x}-\int_{s_{\varepsilon}} \mathcal{S}_{k \rho \lambda} \mathfrak{u}_{\lambda, \delta}(y)\left(x_{\delta}-y_{\delta}\right) \mathrm{d} s_{x}\right\}=0 .
\end{array}
$$

Observe that the second integral in the first line and the first integral in the second line are equal to zero if we take their limits. The value of the last integral in the second line can be manipulated into the form

$$
\lim _{\varepsilon \rightarrow 0}\left\{\int_{s_{\varepsilon}} \mathcal{S}_{k \rho \lambda} \mathrm{d} s_{x} \mathfrak{u}_{\lambda}(y)\right\}=\lim _{\varepsilon \rightarrow 0}\left\{\frac{b_{k \rho \lambda}(y)}{\varepsilon} \mathfrak{u}_{\lambda}(y)\right\} .
$$

This formula clearly shows that the limit of the above integral - due to the fact that the kernel is singular - is unbounded and depends also on the value of $b_{\kappa \rho \lambda}(y)$. However, if the source point $y$ is an internal point, then $b_{\kappa \rho \lambda}(y)=0$ - the proof is omitted here. Therefore, if we take the limit of the last two integrals in (3.7), we have

$$
\lim _{\varepsilon \rightarrow 0}\left\{\int_{s_{\varepsilon}} \mathcal{D}_{k \rho \lambda} u_{\lambda, \pi}(y) \tau_{\pi}(x) \mathrm{d} s_{x}+\int_{s_{\varepsilon}} \mathcal{S}_{k \rho \lambda} \mathfrak{u}_{\lambda, \delta}(y)\left(x_{\delta}-y_{\delta}\right) \mathrm{d} s_{x}\right\}=d_{k \rho \alpha \beta}(y) \mathfrak{u}_{\alpha, \beta}(y),
$$


where $d_{\kappa \rho \alpha \beta}(y) \mathfrak{u}_{\alpha, \beta}=0.5 \mathfrak{u}_{\kappa, \rho}$ if the contour $\mathcal{L}_{o}$ is smooth at the source point $y$. Finally, we obtain

$$
\lim _{\varepsilon \rightarrow 0}\left\{\int_{\mathcal{L}_{\varepsilon}}\left[\mathcal{D}_{k \rho \lambda} \mathfrak{t}_{\lambda}(x)-\mathcal{S}_{k \rho \lambda} \mathfrak{u}_{\lambda}(x)\right] \mathrm{d} s_{x}\right\}=\lim _{\varepsilon \rightarrow 0}\left\{\frac{b_{k \rho \lambda}}{\varepsilon} \mathfrak{u}_{\lambda}(y)\right\}+d_{k \rho \alpha \beta}(y) \mathfrak{u}_{\alpha, \beta}(y) .
$$

or by rearranging it we get

$$
\frac{1}{2} \mathfrak{u}_{k, \rho}(y)+\lim _{\varepsilon \rightarrow 0}\left\{\int_{\mathcal{L}_{o}}\left[\mathcal{S}_{k \rho \lambda} \mathfrak{u}_{\lambda}(x)-\mathcal{D}_{k \rho \lambda} \mathfrak{t}_{\lambda}(x)\right] \mathrm{d} s_{x}+\frac{b_{k \rho \lambda}}{\varepsilon} \mathfrak{u}_{\lambda}(y)\right\}=0 .
$$

Let us introduce the following notations:

$$
\int_{\mathcal{L}_{o}} \mathcal{D}_{k \rho \lambda} \mathfrak{t}_{\lambda}(x) \mathrm{d} s_{x}=\lim _{\varepsilon \rightarrow 0} \int_{\mathcal{L}_{o}} \mathcal{D}_{k \rho \lambda} \mathfrak{t}_{\lambda}(x) \mathrm{d} s_{x}
$$

and

$$
f_{\mathcal{L}_{o}} \mathcal{S}_{k \rho \lambda} \mathfrak{u}_{\lambda}(x) \mathrm{d} s_{x}=\lim _{\varepsilon \rightarrow 0}\left\{\int_{\mathcal{L}_{o}} \mathcal{S}_{k \rho \lambda} \mathfrak{u}_{\lambda}(x) \mathrm{d} s_{x}+\frac{b_{k \rho \lambda}}{\varepsilon} \mathfrak{u}_{\lambda}(y)\right\} .
$$

Making use of the notations introduced, equation (3.11) can be rewritten in the same form as in the primal system of elasticity:

$$
\frac{1}{2} \mathfrak{u}_{k, \rho}(y)=\int_{\mathcal{L}_{o}} \mathcal{D}_{k \rho \lambda} \mathfrak{t}_{\lambda}(x) \mathrm{d} s_{x}-\int_{\mathcal{L}_{o}} \mathcal{S}_{k \rho \lambda} \mathfrak{u}_{\lambda}(x) \mathrm{d} s_{x} .
$$

Assume further that the contour $\mathcal{L}_{o}$ is divided into $n_{b e}$ boundary elements, which have three nodes and are denoted by $\mathcal{L}_{e}$. With the previous notations, the corresponding formula for computing the elements of the stress tensor at the boundary point $y$ is rewritten into this form:

$$
\begin{aligned}
t_{\psi \kappa}(y)=\epsilon_{\psi \rho 3} \mathfrak{u}_{\kappa, \rho}(y)=2 \epsilon_{\psi \rho 3} & \left\{\sum_{e=1}^{n_{b e}} \int_{\mathcal{L}_{e}} \mathcal{D}_{\kappa \rho \lambda}(x, y) \mathfrak{t}_{\lambda}^{e} \mathrm{~d} s_{x}-\right. \\
& \left.-\sum_{e=1, e \neq m}^{n_{b e}} \int_{\mathcal{L}_{e}} \mathcal{S}_{\kappa \rho \lambda}(x, y) \mathfrak{u}_{\lambda}^{e} \mathrm{~d} s_{x}-I_{\kappa \rho \lambda}^{m} \mathfrak{u}_{\lambda}^{m}\right\} .
\end{aligned}
$$

Here the upper index $e$ is the number of the boundary element considered. The element that contains the source point $y$ is denoted by the upper index $m . I_{\kappa \rho j}^{m}$ stands for the line integral with the hypersingular kernel.

\section{NUMERICAL EVALUATION OF THE HYPERSINGULAR INTEGRAL}

Assume that the nodal values of the variables are known from the solution of the direct boundary integral equation method. The nodal values $\mathfrak{u}_{\lambda}^{a}$ and $\mathfrak{t}_{\lambda}^{a}(a=1, \ldots, 3)$, which belong to element $e$ and local node number $a$ are given in the matrix form

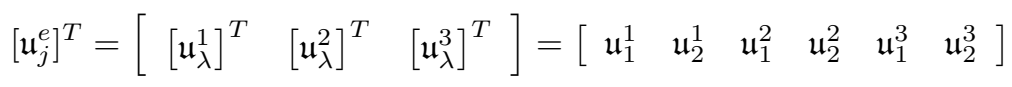

and

$$
\left[\mathfrak{t}_{j}^{e}\right]^{T}=\left[\begin{array}{llllll}
\mathfrak{t}_{1}^{1} & \mathfrak{t}_{2}^{1} & \mathfrak{t}_{1}^{2} & \mathfrak{t}_{2}^{2} & \mathfrak{t}_{1}^{3} & \mathfrak{t}_{2}^{3}
\end{array}\right]
$$


on the $e$-th element $(j=1, \ldots, 6)$. It is obvious that the approximations of the vectors $\mathfrak{u}_{\lambda}$ and $\mathfrak{t}_{\lambda}$ on the $e$-th element take the form:

$$
\mathfrak{u}_{\lambda}=N_{\lambda j}(\xi) \mathfrak{u}_{j}^{e}, \quad \mathfrak{t}_{\lambda}=N_{\lambda j}(\xi) \mathfrak{t}_{j}^{e},
$$

in which $e$ identifies the element and

$$
\left[N_{\lambda j}(\xi)\right]=\left[\begin{array}{cccccc}
N^{1}(\xi) & 0 & N^{2}(\xi) & 0 & N^{3}(\xi) & 0 \\
0 & N^{1}(\xi) & 0 & N^{2}(\xi) & 0 & N^{3}(\xi)
\end{array}\right]
$$

is the shape function matrix constituted by the quadratic Lagrange polynomials

$$
N^{1}(\xi)=\frac{1}{2} \xi(\xi-1), \quad N^{2}(\xi)=1-\xi^{2}, \quad N^{3}(\xi)=\frac{1}{2} \xi(\xi+1)
$$

that map the element onto the interval $\xi \in[-1,1]$ - isoparametric approximation. Making use of approximations (4.3) in (3.15), the stress tensor $t_{\psi \kappa}$ at the boundary point is calculated as follows:

$$
\begin{aligned}
t_{\psi \kappa}(\eta)=2 \epsilon_{\psi \rho 3}\left\{\sum_{e=1}^{n_{b e}} \int_{\mathcal{R}^{e}} \mathcal{D}_{\kappa \rho \lambda}(\xi, \eta) N_{\lambda j}(\xi) J(\xi) \mathrm{d} \xi \mathfrak{t}_{j}^{e}-\right. \\
\left.\quad-\sum_{e=1, e \neq m}^{n_{b e}} \int_{\mathcal{R}^{e}} \mathcal{S}_{\kappa \rho \lambda}(\xi, \eta) N_{\lambda j}(\xi) J(\xi) \mathrm{d} \xi \mathfrak{u}_{j}^{e}-I_{\kappa \rho j}^{m} \mathfrak{u}_{j}^{m}\right\} .
\end{aligned}
$$

Here $J(\xi)$ is the Jacobian, $\mathcal{R}^{e}$ is the map of $\mathcal{L}_{e}$ on the interval $[-1,1]$. The meaning of $\mathcal{R}_{\varepsilon}^{m}$ can be read off from Figure 3 which also shows the map of $\mathcal{L}_{e}$ on the axis $\xi$.

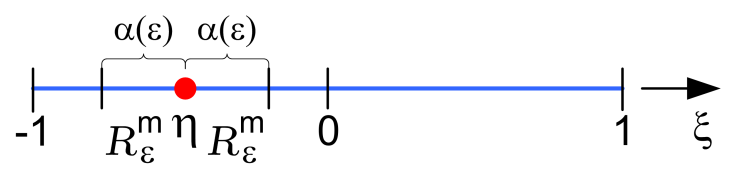

Figure 3. The interval $\mathcal{R}_{\varepsilon}^{m}$

The length of $\mathcal{R}_{\varepsilon}^{m}$ is denoted by $\alpha(\varepsilon)$, while $\eta$ is the image of the source point $y$ on this interval. The line integral containing the hypersingularity can be given in the following form:

$$
I_{\kappa \rho j}^{m}=\int_{\mathcal{R}^{m}} \mathcal{S}_{k \rho \lambda}(\xi, \eta) N_{\lambda j}(\xi) J(\xi) \mathrm{d} \xi=\int_{\mathcal{R}^{m}} F_{\kappa \rho j}^{m}(\xi, \eta) \mathrm{d} \xi .
$$

To determine the power series of $\mathcal{S}_{\kappa \rho \lambda}(\xi, \eta)$ in $\xi$, we have to utilize the equations

$$
\frac{r_{\lambda}}{R}=\frac{A_{\lambda}}{A} \operatorname{sgn}(\delta)+\left[\frac{B_{\lambda}}{A}-\frac{A_{\lambda} C}{A^{3}}\right] \frac{\delta}{\operatorname{sgn}(\delta)}+O\left(\delta^{2}\right)
$$

and

$$
\frac{1}{R^{2}}=\frac{1}{A^{2} \delta^{2}}-2 \frac{C}{A^{4} \delta}+O(1)
$$

in which $\delta=\xi-\eta$. The constants $A_{\lambda}$ and $B_{\lambda}$ are derived from the first two terms of the Taylor series of the position vector $r_{\lambda}$ in the vicinity of the source point: 


$$
r_{\lambda}=x_{\lambda}(\xi)-y_{\lambda}(\eta)=0+\underbrace{\left.\frac{\partial x_{\lambda}}{\partial \xi}\right|_{\xi=\eta}}_{A_{\lambda}} \underbrace{\xi-\eta}_{\delta}+\underbrace{\left.\frac{1}{2 !} \frac{\partial^{2} x_{\lambda}}{\partial \xi^{2}}\right|_{\xi=\eta}}_{\begin{array}{c}
B_{\lambda} \\
=A_{\lambda} \delta+B_{\lambda} \delta^{2}+O\left(\delta^{3}\right),
\end{array}}(\xi-\eta)^{2}+\ldots=
$$

where

$$
A_{\lambda}=\left(x_{\lambda}^{1}-2 x_{\lambda}^{2}+x_{\lambda}^{3}\right) \eta+\frac{1}{2}\left(x_{\lambda}^{3}-x_{\lambda}^{1}\right), \quad B_{\lambda}=\frac{1}{2}\left(x_{\lambda}^{1}-2 x_{\lambda}^{2}+x_{\lambda}^{3}\right)
$$

are constants at $\eta . A=\sqrt{\sum A_{\lambda}^{2}}$ and $C=\sum A_{\lambda} B_{\lambda}$. The power series of the shape function matrix is then

$$
\begin{aligned}
N_{\lambda j}(\xi)=\left.N_{\lambda j}(\xi)\right|_{\xi=\eta}+\left.\frac{\mathrm{d}}{\mathrm{d} \xi} N_{\lambda j}(\xi)\right|_{\xi=\eta}(\xi & -\eta)+O\left(\delta^{2}\right)= \\
& ={ }^{0} N_{\lambda j}(\eta)+{ }^{1} N_{\lambda j}(\eta) \delta+O\left(\delta^{2}\right)
\end{aligned}
$$

in which the elements are given by

$$
\begin{aligned}
& { }^{0} N^{1}(\eta)=\frac{1}{2} \eta(\eta-1), \quad{ }^{0} N^{2}(\eta)=1-\eta^{2}, \quad{ }^{0} N^{3}(\eta)=\frac{1}{2} \eta(\eta+1) \\
& { }^{1} N^{1}(\eta)=\frac{1}{2}(\eta-1), \quad{ }^{1} N^{2}(\eta)=-2 \eta, \quad{ }^{1} N^{3}(\eta)=\frac{1}{2}(\eta+1) .
\end{aligned}
$$

The product of the Jacobian and the vector $n_{\rho}$ can also be given in the following forms:

$$
\begin{aligned}
& J_{1}(\xi)=n_{1} J(\xi)=A_{2}+2 B_{2} \delta+O\left(\delta^{2}\right), \\
& J_{2}(\xi)=n_{2} J(\xi)=-A_{1}-2 B_{1} \delta+O\left(\delta^{2}\right) .
\end{aligned}
$$

After substituting power series $44.8-(4.16)$ into integral $\sqrt{4.7}$, the integrand $F_{\kappa \rho j}^{m}(\xi, \eta)$ can be manipulated in a Laurent series in the vicinity of the source point $\eta$ :

$$
F_{\kappa \rho j}^{m}(\xi, \eta)=\frac{{ }^{2} F_{\kappa \rho j}^{m}(\eta)}{(\xi-\eta)^{2}}+\frac{{ }^{-1} F_{\kappa \rho j}^{m}(\eta)}{\xi-\eta}+O(1),
$$

where ${ }^{-1} F_{\kappa \rho j}^{m}(\eta)$ and ${ }^{-2} F_{\kappa \rho j}^{m}(\eta)$ are constant values at a fixed $\eta \in(-1,1)$. Subtracting the first two terms of the power series from $F_{\kappa \rho j}^{m}(\xi, \eta)$ in equation (4.7) and investigating the terms obtained separately, three line integrals are resulted:

$$
\begin{gathered}
I_{\kappa \rho j}^{m}=\lim _{\varepsilon \rightarrow 0} \int_{\mathcal{R}^{m}-2 \mathcal{R}_{\varepsilon}^{m}}\left\{F_{\kappa \rho j}^{m}(\xi, \eta)-\left[\frac{{ }^{-2} F_{\kappa \rho j}^{m}(\eta)}{(\xi-\eta)^{2}}+\frac{{ }^{-1} F_{\kappa \rho j}^{m}(\eta)}{\xi-\eta}\right]\right\} \mathrm{d} \xi+ \\
+\lim _{\varepsilon \rightarrow 0} \int_{\mathcal{R}^{m}-2 \mathcal{R}_{\varepsilon}^{m}} \frac{-2 F_{\kappa \rho j}^{m}(\eta)}{(\xi-\eta)^{2}} \mathrm{~d} \xi+\lim _{\varepsilon \rightarrow 0} \int_{\mathcal{R}^{m}-2 \mathcal{R}_{\varepsilon}^{m}} \frac{-1 F_{\kappa \rho j}^{m}(\eta)}{\xi-\eta} \mathrm{d} \xi={ }^{0} I_{\kappa \rho j}^{m}+{ }^{-2} I_{\kappa \rho j}^{m}+{ }^{-1} I_{\kappa \rho j}^{m} .
\end{gathered}
$$

Observe that the integrand of ${ }^{0} I_{\kappa \rho j}^{m}$ is a polynomial function without singular terms. Its limit therefore assumes the following form:

$$
{ }^{0} I_{\kappa \rho j}^{m}=\int_{-1}^{1}\left\{F_{\kappa \rho \lambda}(\xi, \eta)-\left[\frac{{ }^{-2} F_{\kappa \rho j}^{m}(\eta)}{(\xi-\eta)^{2}}+\frac{{ }^{-1} F_{\kappa \rho j}^{m}(\eta)}{\xi-\eta}\right]\right\} \mathrm{d} \xi .
$$


This integral is regular and can be calculated numerically by using the standard integration techniques. We have chosen a 14 node Gaussian quadrature rule for the interval $[-1,1]$. The second and third terms on the right side of 4.18 can be determined analytically. To this end we have to use the following expansion of $\alpha$ - see Figure 3 - in the powers of $\varepsilon$ :

$$
\alpha(\varepsilon)=\frac{\varepsilon}{A}-\frac{C}{A^{4}} \varepsilon^{2} \operatorname{sgn}(\alpha)+O\left(\varepsilon^{3}\right) .
$$

After dividing $\mathcal{R}^{m}$ into two subintervals $[-1,-\alpha]$ and $[\alpha, 1]$ at the singular point $\eta$ and making use of series 4.20 we can determine the limit of the last two integrals in equation 4.18. After some hand calculations, we obtain the following analytical result

$$
\begin{array}{r}
{ }^{-1} I_{\kappa \rho j}^{m}=\lim _{\varepsilon \rightarrow 0^{-}}\left\{\int_{-1}^{\eta-\alpha} \frac{{ }^{-1} F_{\kappa \rho j}^{m}(\eta)}{\xi-\eta} \mathrm{d} \xi\right\}+\lim _{\varepsilon \rightarrow 0^{+}}\left\{\int_{\eta+\alpha}^{1} \frac{{ }^{-1} F_{\kappa \rho j}^{m}(\eta)}{\xi-\eta} \mathrm{d} \xi\right\}= \\
={ }^{-1} F_{\kappa \rho j}^{m}(\eta) \ln \left|\frac{1-\eta}{-1-\eta}\right| .
\end{array}
$$

Likewise, the last integral in equation 4.18 can also be given in a closed form:

$$
\begin{aligned}
& { }^{-2} I_{\kappa \rho j}^{m}=\lim _{\varepsilon \rightarrow 0^{-}}\left\{\int_{-1}^{\eta-\alpha} \frac{{ }^{-2} F_{\kappa \rho j}^{m}(\eta)}{(\xi-\eta)^{2}} \mathrm{~d} \xi\right\}+\lim _{\varepsilon \rightarrow 0^{+}}\left\{\int_{\eta+\alpha}^{1} \frac{-2 F_{\kappa \rho j}^{m}(\eta)}{(\xi-\eta)^{2}} \mathrm{~d} \xi\right\}= \\
& ={ }^{-2} F_{\kappa \rho j}^{m}(\eta)\left(\frac{1}{-1-\eta}-\frac{1}{1-\eta}\right) \text {. }
\end{aligned}
$$

Consequently, formulae 4.19, 4.21 and 4.22 can be used to determine the value of the hypersingular integrals over the element containing the hypersingularity.

It is obvious that these integrals can easily be calculated and thus they can given in closed form. After collecting partial results, the final formula for the evaluation of the hypersingular integral is given by

$$
\begin{aligned}
I_{\kappa \rho j}^{m}=\int_{-1}^{1} F_{\kappa \rho j}^{m}(\xi, \eta) & -\left[\frac{{ }^{-2} F_{\kappa \rho j}^{m}(\eta)}{(\xi-\eta)^{2}}+\frac{{ }^{-1} F_{\kappa \rho j}^{m}(\eta)}{\xi-\eta}\right] \mathrm{d} \xi+ \\
& +{ }^{-2} F_{\kappa \rho j}^{m}(\eta)\left(-\frac{1}{1-\eta}+\frac{1}{-1-\eta}\right)+{ }^{-1} F_{\kappa \rho j}^{m} \ln \left|\frac{1-\eta}{-1-\eta}\right|,
\end{aligned}
$$

where $\eta \in(-1,1)$. In those cases where $\eta=-1$ or $\eta=1$ the previous derivation leads to the following formula:

$$
\begin{aligned}
I_{\kappa \rho j}=\sum_{m=1}^{2}\left\{\int_{-1}^{1} F_{\kappa \rho \lambda}^{m}(\xi, \eta)-\left[\frac{{ }^{-2} F_{\kappa \rho j}^{m}(\eta)}{(\xi-\eta)^{2}}+\frac{{ }^{-1} F_{\kappa \rho j}^{m}(\eta)}{\xi-\eta}\right] \mathrm{d} \xi-\right. \\
\left.\quad-{ }^{-2} F_{\kappa \rho j}^{m}(\eta)\left(\frac{\gamma_{m}}{\beta_{m}^{2}} \operatorname{sgn}(\delta)+\frac{1}{2}\right)+{ }^{-1} F_{\kappa \rho j}^{m} \ln \left|\frac{2}{\beta_{m}}\right| \operatorname{sgn}(\delta)\right\} .
\end{aligned}
$$


The calculation program, written in Fortran90, has been developed for the numerical solution using the formulae of 6 ] and equations (4.23), 4.24.

\section{Numerical EXAMPLES}

Problem 1. We shall consider a circular region with radius $R=10 \mathrm{~mm}$. The material properties are $\mu=8 \cdot 10^{4} \mathrm{MPa}, \nu=0.3$.

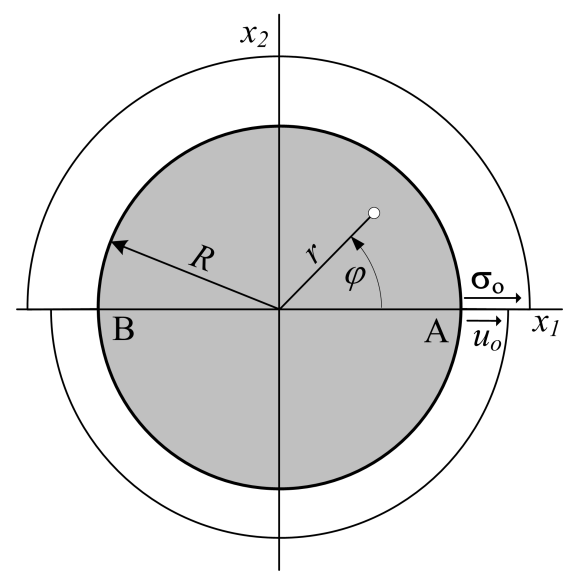

Figure 4. A circular region

On the arc $A B$ of the contour for which the angle $\varphi \in[0, \pi]$ the normal stress is $\sigma_{o}=100 \mathrm{MPa}$ and there is no shear stress. On the $\operatorname{arc} B A$ of the contour $u_{o}=(1-2 \nu) \sigma_{o} r_{o} / 2 \mu$ is the radial displacement and there is no displacement in the circumferential direction - see Figure 4 . One can check with ease that these solutions determine a homogenous state of stress. At the points of the region $\mathcal{A}_{i}$ the exact solutions for the stresses are as follows:

$$
\sigma_{1}=\sigma_{2}=\sigma_{\mathrm{o}}=100[\mathrm{MPa}], \quad \tau_{12}=\tau_{21}=0 .
$$

Table 1 below shows how the accuracy of numerical results at a boundary point is increased if we increase the number of boundary elements $n_{b e}$.

Table 1: Solutions for stress components

\begin{tabular}{|c|c|c|c|}
\hline$n_{b e}$ & $\sigma_{1}[\mathrm{MPa}]$ & $\tau_{12}=\tau_{21}[\mathrm{MPa}]$ & $\sigma_{2}[\mathrm{MPa}]$ \\
\hline 8 & 99.86512 & $-6.04096 \cdot 10^{-3}$ & 99.88720 \\
\hline 16 & 99.98509 & $-6.17259 \cdot 10^{-4}$ & 99.98576 \\
\hline 32 & 99.99821 & $-4.42785 \cdot 10^{-5}$ & 99.99823 \\
\hline 64 & 99.99978 & $-2.74219 \cdot 10^{-6}$ & 99.99957 \\
\hline
\end{tabular}

Problem 2. Though the contour $\mathcal{L}_{o}$ and the material are the same as in the previous examples the region under consideration is the outer one, for which a constant stress state $\sigma_{1}(\infty)=100 \mathrm{MPa}, \tau_{12}(\infty)=\tau_{21}(\infty)=\sigma_{2}(\infty)=0$ is prescribed at infinity. The inner boundary is free of displacement constraints. 


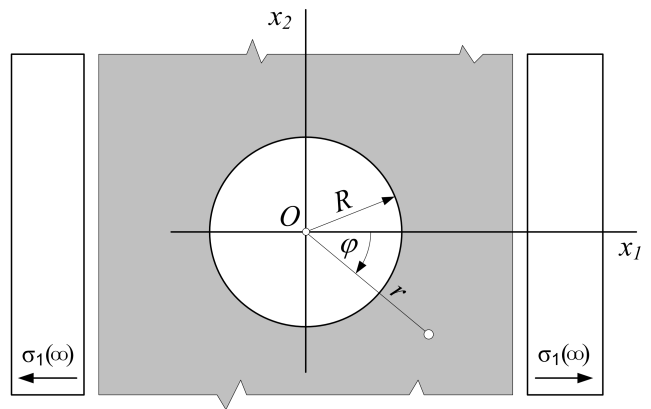

Figure 5. Outer region bounded by a circle

It is well known that the formulae

$$
\begin{array}{r}
\sigma_{r}=\frac{\sigma_{1}(\infty)}{2}\left[\left(1-\frac{r_{o}^{2}}{r^{2}}\right)+\left(1+\frac{3 r_{o}^{4}}{r^{4}}-\frac{4 r_{o}^{2}}{r^{2}}\right) \cos 2 \varphi\right], \\
\sigma_{\varphi}=\frac{\sigma_{1}(\infty)}{2}\left[\left(1+\frac{r_{o}^{2}}{r^{2}}\right)-\left(1+\frac{3 r_{o}^{4}}{r^{4}}\right) \cos 2 \varphi\right], \\
\tau_{r \varphi}=\frac{\sigma_{1}(\infty)}{2}\left[\left(1-\frac{3 r_{o}^{4}}{r^{4}}+\frac{2 r_{o}^{2}}{r^{2}}\right) \sin 2 \varphi\right] .
\end{array}
$$

written in polar coordinates give the exact solution to this problem - see 8 or $[9]$. The exact solution on the whole boundary curve is computed from (5.1) in the coordinate system $\left(x_{1}, x_{2}\right)$ :

$$
\begin{array}{r}
\sigma_{1}=\sigma_{1}(\infty)[1-2 \cos 2 \varphi] \sin ^{2} \varphi, \\
\sigma_{2}=\sigma_{1}(\infty)[1-2 \cos 2 \varphi] \cos ^{2} \varphi, \\
\tau_{12}=\tau_{21}=\sigma_{1}(\infty)[1-2 \cos 2 \varphi] \sin \varphi \cos \varphi
\end{array}
$$

The contour was divided into 16 equidistant elements. The diagrams below show the stresses as function $\varphi$ along the circle in clockwise direction.

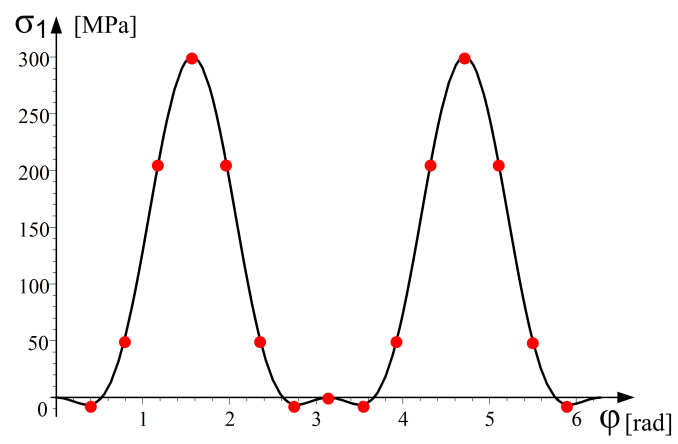

Figure 6. Exact and numerical solution $-\sigma_{1}$ as function $\varphi$ 


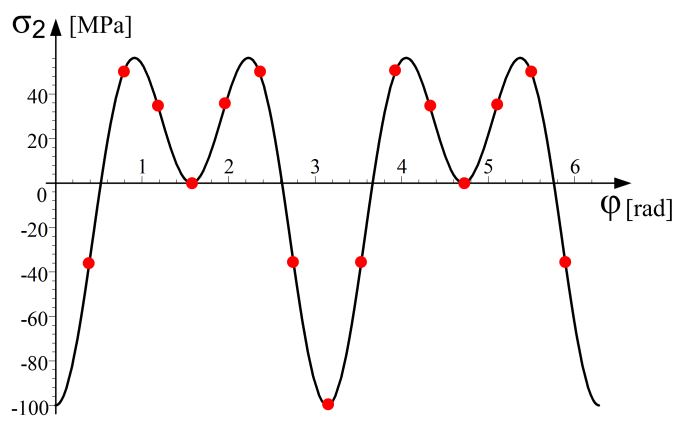

Figure 7. Exact and numerical solution $-\sigma_{2}$ as function $\varphi$

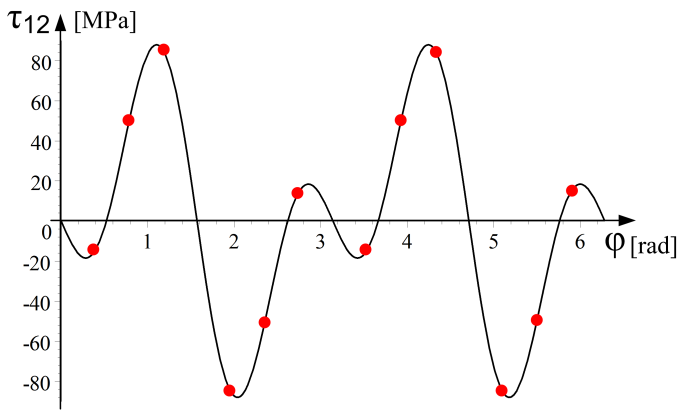

Figure 8. Exact and numerical solution $-\tau_{12}$ as function $\varphi$

Table 2 below represents the numerical results obtained at various boundary points.

Table 2: Solutions for stress components

\begin{tabular}{|c|c|c|c|c|c|c|}
\hline$\varphi$ & \multicolumn{2}{|c|}{$\sigma_{1}[\mathrm{MPa}]$} & \multicolumn{2}{c|}{$\tau_{12}=\tau_{21}[\mathrm{MPa}]$} & \multicolumn{2}{c|}{$\sigma_{2}[\mathrm{MPa}]$} \\
\hline$\pi / 8$ & -6.085 & -6.066 & -14.679 & -14.645 & -35.346 & -35.355 \\
\hline$\pi / 4$ & 50.026 & 50.00 & 50.047 & 50.00 & 50.072 & 50.00 \\
\hline $3 \pi / 8$ & 206.169 & 206.07 & 85.457 & 85.355 & 35.353 & 35.355 \\
\hline$\pi / 2$ & 300.196 & 300.00 & 0.00 & 0.00 & -0.072 & 0.00 \\
\hline
\end{tabular}

The numerical results show a good agreement with the analytical results that appear in the second columns.

\section{Concluding REmarks}

This paper is concerned with a hypersingular boundary integral formulation in the dual system of plane elasticity. Our main objective was to present a method by which the stress components on the boundary can be computed with high accuracy both in the plane region and on its boundary. We have developed the hypersingular boundary 
integral equation (HBIE) method in the dual system of plane elasticity providing that (i) the region under consideration is a simply-connected one, (ii) there are no body forces, (iii) the contour consists of two arcs on which tractions and displacements are prescribed, respectively. The line of thought is based on a technique developed by Guiggiani et al. 11 for the primal system of plane elasticity.

It has been proven that the formulae for calculating the stresses on the boundary can be given in closed forms. The hypersingular integrals in these formulae can be resolved into two parts. The first one should be computed numerically - this computation results in, however, a very small numerical error. The second part can be given in a closed form. Consequently, in contrast to the conventional stress calculation, which uses polynomial approximations for the derivatives and thus might lead to significant errors both in the formalism and in the numerical results in the vicinity of the boundary, the stress calculation method suggested in this paper in the dual system is much more reliable and accurate due to the way we handle the hypersingular integrals.

A program has been developed in Fortran 90 for the numerical computations. The results shown demonstrate that the accuracy of the computations is increased in the dual formulations if we use hypersingular integral equations.

Acknowledgements. The described work was carried out as part of the TÁMOP-4.2.1.B10/2/KONV-2010-0001 project in the framework of the New Hungarian Development Plan. The realization of this project is supported by the European Union, co-financed by the European Social Fund.

\section{REFERENCES}

1. Guiggiani, M., Krishnasamy, G., Rizzo, F. J., and Randolphi, T. J.: Hypersingular boundary integral equations: a new approach to their numerical treatment. IABEM Symposium, 1990.

2. Guiggiani, M.: Direct evluation of hypersingular integrals in 2D BEM, (7th GAMM Seminar on Numerical techniques for Boundary Element Method, Kiel, Germany, 1991). In W. Hackbusch (ed.), Notes in Numerical Fluid Mechanics, 1992, pp. 23-34.

3. Hildenbrand, J. and Kunn, G.: Numerical computation of hypersingular integrals and application to the boundary integral equation for the stress tensor. Engineering Analysis with Boundary Elements, 10, (1992), 209-217.

4. Guiggiani, M.: Hypersingular formulation for boundary stress evaluation. Engineering Analysis with Boundary Elements, 13(2), (1994), 169-179.

5. SzeIDL, G.: Dual problems of continuum mechanics (derivation of defining equations, single valuedness of mixed boundary value problems, boundary element method for plane problems). Habilitation Booklets of the Miskolc University (in Hungarian), Faculty of Mechanical Engineering, University of Miskolc, Department of Mechanics, 1997.

6. SzeidL, G.: Boundary integral equations for plane problems in terms of stress functions of order one. Journal of Computational and Applied Mechanics, 2(2), (2001), 237-261.

7. SzeidL, G.: Problems of Continuum Mechanics in a Dual Formulation (Derivation of the definig equations, Single valuedness of mixed boundary value problems, Boundary element 
method for plane problems). Thesis for the degree of Doctor of Science awarded by the Hungarian Academy of Sciences (in Hungarian), 2004.

8. Timoshenko, S. and Goodier, J. N.: Theory of Elasticity. McGraw-Hill, 1951.

9. Muskhelisvili, I. I.: Some Fundamental Problems of Mathematical Theory of Elasticity. Publisher NAUKA, 1966.

\section{Appendix A.}

A.1. Derivatives of the fundamental solution of order two. Making use of equation 2.3. we can determine the derivatives $\mathcal{S}_{k \rho \lambda}=\mathfrak{T}_{\kappa \lambda, \rho}$. The results are presented below:

$$
\begin{aligned}
& \mathcal{S}_{111}=\mathfrak{D}(R)\left\{\frac{1}{R^{2}}\left[n_{1} r_{2}\left(16 \frac{r_{1}^{2} r_{2}}{R^{2}}+4(1-2 \nu) r_{2}\right)+n_{2} r_{1}\left(16 \frac{r_{2}^{3}}{R^{2}}-4(3-2 \nu) r_{2}\right)\right]-\right. \\
& \left.-\left[n_{1}\left(4 \frac{r_{1}^{2}}{R^{2}}+2(1-2 \nu)\right)\right]\right\} \\
& \mathcal{S}_{112}=\mathfrak{D}(R)\left\{-\frac{1}{R^{2}} n_{1} r_{2}\left(16 \frac{r_{1}^{3}}{R^{2}}-4(3-2 \nu) r_{1}\right)-\frac{1}{R^{2}} n_{2} r_{1}\left(16 \frac{r_{1} r_{2}^{2}}{R^{2}}+4(1-2 \nu) r_{1}\right)+\right. \\
& \left.+n_{2}\left(4 \frac{r_{2}^{2}}{R^{2}}+2(1-2 \nu)\right)\right\} \\
& \mathcal{S}_{211}=\mathfrak{D}(R)\left\{-\frac{1}{R^{2}} n_{1} r_{2}\left(16 \frac{r_{1}^{3}}{R^{2}}-4(1+2 \nu) r_{1}\right)-\frac{1}{R^{2}} n_{2} r_{1}\left(16 \frac{r_{1}^{3}}{R^{2}}-4(1-2 \nu) r_{1}\right)+\right. \\
& \left.+n_{2}\left(4 \frac{r_{2}^{2}}{R^{2}}-2(1-2 \nu)\right)\right\} \\
& \mathcal{S}_{212}=\mathfrak{D}(R)\left\{\frac{1}{R^{2}} n_{2} r_{2}\left(16 \frac{r_{1}^{3}}{R^{2}}-4(5-2 \nu) r_{1}\right)+\frac{1}{R^{2}} n_{1} r_{1}\left(16 \frac{r_{1}^{3}}{R^{2}}-4(5-2 \nu) r_{1}\right)-\right. \\
& \left.-n_{1}\left(4 \frac{r_{1}^{2}}{R^{2}}-2(3-2 \nu)\right)\right\} \\
& \mathcal{S}_{121}=\mathfrak{D}(R)\left\{\frac{1}{R^{2}} n_{1} r_{1}\left(16 \frac{r_{2}^{3}}{R^{2}}-4(5-2 \nu) r_{2}\right)+\frac{1}{R^{2}} n_{2} r_{2}\left(16 \frac{r_{2}^{3}}{R^{2}}-4(5-2 \nu) r_{2}\right)-\right. \\
& \left.-n_{2}\left(4 \frac{r_{2}^{2}}{R^{2}}-2(3-2 \nu)\right)\right\} \\
& \mathcal{S}_{122}=\mathfrak{D}(R)\left\{-\frac{1}{R^{2}} n_{2} r_{1}\left(16 \frac{r_{2}^{3}}{R^{2}}-4(1+2 \nu) r_{2}\right)-\frac{1}{R^{2}} n_{1} r_{2}\left(16 \frac{r_{1}^{2} r_{2}}{R^{2}}-4(1-2 \nu) r_{2}\right)+\right. \\
& \left.+n_{1}\left(4 \frac{r_{1}^{2}}{R^{2}}-2(1-2 \nu)\right)\right\} \\
& \mathcal{S}_{221}=\mathfrak{D}(R)\left\{\frac{1}{R^{2}}\left[-n_{1} r_{2}\left(16 \frac{r_{1}^{2} r_{2}}{R^{2}}+4(1-2 \nu) r_{2}\right)-n_{2} r_{1}\left(16 \frac{r_{2}^{3}}{R^{2}}-4(3-2 \nu) r_{2}\right)\right]+\right. \\
& \left.+n_{1}\left(4 \frac{r_{1}^{2}}{R^{2}}+2(1-2 \nu)\right)\right\}
\end{aligned}
$$




$$
\begin{array}{r}
\mathcal{S}_{222}=\mathfrak{D}(R)\left\{\frac{1}{R^{2}}\left[n_{1} r_{2}\left(16 \frac{r_{1}^{3}}{R^{2}}-4(3-2 \nu) r_{1}\right)+n_{2} r_{1}\left(16 \frac{r_{1} r_{2}^{2}}{R^{2}}+4(1-2 \nu) r_{1}\right)\right]-\right. \\
\left.-n_{2}\left(4 \frac{r_{2}^{2}}{R^{2}}+2(1-2 \nu)\right)\right\}
\end{array}
$$

where $\mathfrak{D}(R)=1 / 8 \pi(1-\nu) R^{2}$ and the normal $n_{\rho}$ is taken at the point $x$.

A.2. Series expansion of the hypersingular integrand $F_{\kappa \rho j}^{m}(\xi, \eta)$. Applying the power series 4.8 4.11, we can determine the series expansion of the matrix $\mathcal{S}_{\kappa \rho \lambda}$ at $\eta$ as follows:

$$
\mathcal{S}_{\kappa \rho \lambda}(\xi, \eta) J(\xi)={ }^{0} a_{\kappa \rho \lambda}+{ }^{1} a_{\kappa \rho \lambda} \delta+O\left(\delta^{2}\right),
$$

in which

$$
{ }^{0} a_{111}=\frac{A_{2}}{A^{2}}\left(4 A_{1}^{2}+\left(2 A_{1}^{2}+2 A_{2}^{2}-A^{2}\right) \mathfrak{K}_{3}\right)
$$

$$
\begin{aligned}
{ }^{1} a_{111}= & -\left(\frac{2}{A^{4}} 4 C A_{1}^{2} A_{2}+8 A_{1} A_{2}^{3} B_{1}-8 A_{1}^{2} A_{2}^{2} B_{2}-8 A^{2} A_{1} A_{2} B_{1}+\right. \\
& \left.+\left(A^{4} B_{2}+2 C A_{2}^{3}+2 C A_{1}^{2} A_{2}-A^{2} A_{1}^{2} B_{2}-4 A^{2} A_{2}^{2} B_{2}-3 A^{2} A_{1} A_{2} B_{1}\right) \mathfrak{K}_{3}\right) \\
0{ }^{0} a_{112}= & \frac{A_{1}}{A^{2}}\left(4 A_{2}^{2}+\left(2 A_{1}^{2}+2 A_{2}^{2}-A^{2}\right) \mathfrak{K}_{3}\right) \\
{ }^{1} a_{112}= & -\frac{2}{A^{4}}\left(4 C A_{1} A_{2}^{2}+8 A_{1}^{3} A_{2} B_{2}-8 A_{1}^{2} A_{2}^{2} B_{1}-8 A^{2} A_{1} A_{2} B_{2}+\right. \\
& \left.+\left(A^{4} B_{1}+2 C A_{1}^{3}+2 C A_{1} A_{2}^{2}-4 A^{2} A_{1}^{2} B_{1}-A^{2} A_{2}^{2} B_{1}-3 A^{2} A_{1} A_{2} B_{2}\right) \mathfrak{K}_{3}\right) \\
& { }^{0} a_{211}=\frac{A_{1}}{A^{2}}\left(4 A_{2}^{2}-\left(2 A_{2}^{2}+2 A_{1}^{2}-A^{2}\right) \mathfrak{K}_{3}\right) \\
{ }^{1} a_{211}= & \frac{2}{A^{4}}\left(-4 C A_{1} A_{2}^{2}-8 A_{1}^{3} A_{2} B_{2}+8 A_{1}^{2} A_{2}^{2} B_{1}+8 A^{2} A_{1} A_{2} B_{2}+\right. \\
& \left.+\left(A^{4} B_{1}+2 C A_{1}^{3}+2 C A_{1} A_{2}^{2}-4 A^{2} A_{1}^{2} B_{1}-A^{2} A_{2}^{2} B_{1}-3 A^{2} A_{1} A_{2} B_{2}\right) \mathfrak{K}_{3}\right) \\
& { }^{0} a_{212}=\frac{A_{2}}{A^{2}}\left(4 A^{2}-4 A_{1}^{2}+A_{3}^{2} \mathfrak{K}_{3}\right)
\end{aligned}
$$$$
{ }^{1} a_{212}=\frac{2}{A^{4}}\left(4 A^{4} B_{2}+8 A_{1}^{4} B_{2}+4 C A_{1}^{2} A_{2}-8 A_{1}^{3} A_{2} B_{1}-12 A^{2} A_{1}^{2} B_{2}+4 A^{2} A_{1} A_{2} B_{1}+\right.
$$$$
\left.+\left(A^{4} B_{2}-A^{2} A_{1}^{2} B_{2}+A^{2} A_{1} A_{2} B_{1}\right) \mathfrak{K}_{3}\right)
$$$$
a_{121}^{0}=-\frac{A_{1}}{A^{2}}\left(4 A^{2}-4 A_{2}^{2}+A_{3}^{2} \mathfrak{K}_{3}\right)
$$$$
{ }^{1} a_{121}=-\frac{2}{A^{4}}\left(4 A^{4} B_{1}+8 A_{2}^{4} B_{1}+4 C A_{1} A_{2}^{2}-8 A_{1} A_{2}^{3} B_{2}-12 A^{2} A_{2}^{2} B_{1}+4 A^{2} A_{1} A_{2} B_{2}+\right.
$$$$
\left.+\left(A^{4} B_{1}-A^{2} A_{2}^{2} B_{1}+A^{2} A_{1} A_{2} B_{2}\right) \mathfrak{K}_{3}\right)
$$$$
a_{122}^{0}=-\frac{A_{2}}{A^{2}}\left(4 A_{1}^{2}-\left(2 A_{2}^{2}+2 A_{1}^{2}-A^{2}\right) \mathfrak{K}_{3}\right)
$$$$
{ }^{1} a_{122}=-\frac{2}{A^{4}}\left(-4 C A_{1}^{2} A_{2}-8 A_{1} A_{2}^{3} B_{1}+8 A_{1}^{2} A_{2}^{2} B_{2}+8 A^{2} A_{1} A_{2} B_{1}+\right.
$$$$
\left.+\left(A^{4} B_{2}-A^{2} A_{1}^{2} B_{2}-4 A^{2} A_{2}^{2} B_{2}+2 C A_{2}^{3}+2 C A_{1}^{2} A_{2}-3 A^{2} A_{1} A_{2} B_{1}\right) \mathfrak{K}_{3}\right)
$$$$
{ }^{0} a_{221}=-\frac{A_{2}}{A^{2}}\left(4 A_{1}^{2}+\left(2 A_{1}^{2}+2 A_{2}^{2}-A^{2}\right) \mathfrak{K}_{3}\right)
$$ 


$$
\begin{aligned}
&{ }^{1} a_{221}= \frac{2}{A^{4}}\left(4 C A_{1}^{2} A_{2}+8 A_{1} A_{2}^{3} B_{1}-8 A_{1}^{2} A_{2}^{2} B_{2}-8 A^{2} A_{1} A_{2} B_{1}+\right. \\
&\left.+\left(A^{4} B_{2}+2 C A_{2}^{3}+2 C A_{1}^{2} A_{2}-A^{2} A_{1}^{2} B_{2}-4 A^{2} A_{2}^{2} B_{2}-3 A^{2} A_{1} A_{2} B_{1}\right) \mathfrak{K}_{3}\right) \\
&{ }^{0} a_{222}=-\frac{A_{1}}{A^{2}}\left(4 A_{2}^{2}+\left(2 A_{1}^{2}+2 A_{2}^{2}-A^{2}\right) \mathfrak{K}_{3}\right) \\
&{ }^{1} a_{222}= \\
& \quad \frac{2}{A^{4}}\left(4 C A_{1} A_{2}^{2}+8 A_{1}^{3} A_{2} B_{2}-8 A_{1}^{2} A_{2}^{2} B_{1}-8 A^{2} A_{1} A_{2} B_{2}+\right. \\
&\left.+\left(A^{4} B_{1}+2 C A_{1}^{3}+2 C A_{1} A_{2}^{2}-4 A^{2} A_{1}^{2} B_{1}-A^{2} A_{2}^{2} B_{1}-3 A^{2} A_{1} A_{2} B_{2}\right) \mathfrak{K}_{3}\right)
\end{aligned}
$$

where $\mathfrak{K}_{2}=1 / 8 \pi(1-\nu)$ and $\mathfrak{K}_{3}=2(1-2 \nu)$. In addition, exploiting equations 4.12)-(4.16), the Laurent series of $F_{\kappa \rho j}^{m}(\xi, \eta)$ in accordance with equation (4.17) is given in this form:

$$
\begin{aligned}
& F_{\kappa \rho j}^{m}(\xi, \eta)=\mathcal{S}_{\kappa \rho \lambda}(\xi, \eta) J(\xi) N_{\lambda j}(\xi)=\underbrace{\left[\frac{1}{A^{2} \mathfrak{K}_{2}}{ }^{0} a_{\kappa \rho \lambda}{ }^{0} N_{\lambda j}(\eta)\right]}_{-2 F_{\kappa \rho j}^{m}(\eta)} \delta^{-2}+ \\
& +\underbrace{\left[\left(\frac{1}{A^{2} \mathfrak{K}_{2}}{ }^{1} a_{\kappa \rho \lambda}-\frac{2 C}{A^{4} \mathfrak{K}_{2}}{ }^{0} a_{\kappa \rho \lambda}\right){ }^{0} N_{\lambda j}(\eta)+\frac{1}{A^{2} \mathfrak{K}_{2}}{ }^{0} a_{\kappa \rho \lambda}{ }^{1} N_{\lambda j}(\eta)\right]}_{-1 F_{\kappa \rho j}^{m}(\eta)} \delta^{-1}+O(1)
\end{aligned}
$$

